\title{
Expiratory Upper Airway Obstruction Caused by the Soft Palate during Bag-Mask Ventilation
}

\author{
Charles W. Buffington, Cynthia M. Q. Wells, Ryan J. Soose
}

Departments of Anesthesiology and Otolaryngology, University of Pittsburgh, Pittsburgh, USA.

Email: buffingtoncw@anes.upmc.edu

Received December $20^{\text {th }}, 2011$, revised January $5^{\text {th }}, 2012$, accepted January $16^{\text {th }}, 2012$

\begin{abstract}
Introduction: Expiratory upper airway obstruction during bag-mask ventilation is not well characterized. Methods: An audit was done to assess expiratory obstruction in 90 adult surgical patients undergoing bag-mask ventilation during the induction of general anaesthesia. Results: Clinicians experienced difficulty delivering gas to the lungs when the head was neutral in 52 of 90 patients (58\%; inspiratory obstruction) but this problem was corrected by head tilt and chin lift in all but 2 patients. Clinicians experienced difficulty recovering gas from the lungs when the mouth was held closed under the mask in 30 of the remaining 88 patients (34\%; expiratory obstruction). This problem persisted despite head tilt and chin lift in all but one patient but was uniformly corrected by opening the mouth. Inspection of the soft palate revealed that it was lying on the posterior pharyngeal wall in 27 of 30 patients with expiratory obstruction and that the retropalatal space was patent in 55 of 58 patients without expiratory obstruction $\left(\chi^{2}, \mathrm{P}<0.001\right)$. The clinical predictors of expiratory upper airway obstruction included advanced age, large tongue, and large uvula. Conclusion: Expiratory airway obstruction should be suspected in all cases of difficult mask ventilation that cannot be corrected by head tilt and chin lift. Simply allowing the mouth to open between positive pressure breaths will permit gas to exit the lungs.
\end{abstract}

Keywords: Expiration; Soft Palate; Mask Ventilation

\section{Introduction}

Manual ventilation with a bag and face mask is used during the period between the induction of general anaesthesia and intubation as well as in clinical settings involving resuscitation. Obstruction of the upper airway during inspiration by the tongue or epiglottis complicates bag-mask ventilation in unconscious patients but can usually be corrected by tilting the head backwards and lifting the chin $[1,2]$. Upper airway obstruction during expiration is less well understood, and only brief references to the phenomenon appear in the literature [3,4]. The concept is that gas can be delivered by positive pressure to the lungs via the nose, nasopharynx and pharynx but is unable to reverse course because the soft palate forms a seal with the posterior pharyngeal wall, blocking egress through the nose. If the mask is tightly applied to the face and the mouth held shut, then inspired gas has no expiratory route and "breath stacking" occurs with subsequent positive pressure breaths. This phenomenon is estimated to occur in $5 \%-10 \%$ of patients [5, 6], but the problem has never been formally studied, nor has the imputed mechanism-soft-palate obstructionbeen verified. Careful observation during the course of clinical practice convinced us that the phenomenon was both more wide-spread than previously thought and poorly understood by clinicians at our hospital. In an effort to improve the safety of bag-mask ventilation, we have conducted an audit of patients undergoing general endotracheal anaesthesia with the aim of establishing the incidence, clinical predictors, mechanism, and treatment of expiratory upper airway obstruction.

\section{Materials and Methods}

This audit of bag-mask ventilation during the induction of anaesthesia was approved by the Total Quality Council of Presbyterian University Hospital, Pittsburgh, PA (project \# 622). Because this was an audit of clinical care, patient consent was neither required nor obtained.

Data were collected from 90 randomly selected adults undergoing general endotracheal anaesthesia for surgical procedures at our hospital, a tertiary care center for adults. One of the authors (CWB or CMQW) interviewed each patient before anaesthesia and was present in the operating room during induction of anaesthesia. During the interview, clinical data about the patient's upper airway were collected and a thorough visual examination of the patient's face, neck and airway was done. The view (or not) of the uvula, soft palate and pharynx was catego- 
rized by means of the Friedman Tongue Position scale [7], a modification of the Mallampati score that provides a finer gradation. The tongue was rated "large" if it took up a lot of room in the mouth. The uvula was rated "large" if it was either elongated and pendulous or wide from side to side. The uvula could be seen and rated when the patient was awake in about half of the patients and was examined during anaesthesia in the other half.

Patients were asked if they snored during sleep, and their responses were subjectively categorized as "no”, “a little" or "a lot” based on their report and often, a family member's input. They were asked if anyone had ever told them they might have obstructive sleep apnea (OSA). They were also asked if they had been diagnosed as having OSA and used a continuous positive airway pressure (CPAP) mask during sleep. Patients were asked to puff out their cheeks to check for palato-pharyngeal competence since the velum must close off the retropalatal space effectively to allow air pressure to build up in the mouth. A tape measure was used to determine neck circumference.

Patients were excluded from the audit if they did not demonstrate palato-pharyngeal competence, had tumor or visible anomaly of the upper airway, if they had a tube passing through the nasopharynx, if they were unable to breathe through their nose, or if they had a tracheostomy.

In the operating room, each patient was positioned supine with the head supported by a 2-inch high, donutshaped foam pillow. After general anaesthesia was induced with standard bolus doses of Propofol and a muscle relaxant, bag-mask ventilation with oxygen was attempted with the head in the neutral position. If gas could not be delivered to the lungs, the head was tilted and the chin lifted (without opening the mouth), maneuvers that almost always corrected inspiratory obstruction. Then a single positive pressure breath was given with the lips together and mask tightly applied to the face. The patient was observed for clinical signs of expiratory upper airway obstruction: chest rise but not fall and lack of a $\mathrm{CO}_{2}$ waveform on the capnograph. Several more breaths were given, the chest was observed for continued expansion (breath stacking), and then the mask was lifted and the jaw released. After release, the mouth and lips were observed for fluttering due to forceful egress of gas. A diagnosis of expiratory upper airway obstruction was made if all 3 signs were present: breath stacking plus no expiratory $\mathrm{CO}_{2}$ plus forceful egress of gas from the mouth when the mask was removed.

When the patient was ready for intubation, one of the authors inserted a laryngoscope with a straight blade into the mouth and used it like a tongue blade to carefully push the tongue inferiorly in order to view the soft palate and uvula. The retropalatal space was rated as patent or obstructed based on the presence or absence of a shadow behind the soft palate and the presence or absence of a continuous coating of saliva that included the velum, uvula and posterior pharyngeal wall. In 31 randomlyselected cases, a video laryngoscope (Glidescope, Cobalt AVL Monitor, Verathon.com) was used for intubation after straight blade visualization of the soft palate. In these 31 cases, a 3 - 5 s video clip of the soft palate was obtained during the process. The video laryngoscope's blade was curved, and care was taken to minimize lifting the tongue during the video recording. The video clips were blinded and reviewed by an otolaryngologist (RJS) with experience in soft-palate surgery for OSA who did not take part in the clinical evaluation. The otolaryngologist rated the retropalatal space as patent or obstructed. This extra step was done to check for inaccuracy or bias in ratings done in the operating room.

Statistical Analysis: Categorical variables were coded 1 if present and 0 if not. Body mass index (BMI) was calculated from the patient's height and weight by the standard formula. Age was converted to decades, with the youngest patient (18 years old) lumped with the second decade and two octogenarians lumped into the seventh decade. The data were entered by hand into a computer and SPSS (version 18.0; IBM, Somers, NY) was used for statistical analysis. The audit was designed as a descriptive study and no comparisons were planned, so no power analysis was done. Descriptive statistics were computed. Univariate correlations between variables were computed. Variables that were significantly correlated with expiratory upper airway obstruction were entered into a stepwise, multivariable regression analysis. Chi Square analysis was used to assess the significance of the relationship between the patency of the retropalatal space and the occurrence of expiratory airway obstruction. $\mathrm{P}<0.05$ was considered significant. Data are presented as means $\pm 1 \mathrm{SD}$.

\section{Results}

The patients' age averaged $54 \pm 16$ (range 18 - 85) years old (Table 1). BMI averaged $29 \pm 6$ with a range from 18 to 47 . Twenty nine patients reported that they never snored, 29 that they snored “a little”, and 23 admitted to snoring "a lot”. Nine patients slept alone and were unsure whether they snored or not. Twenty three percent of patients admitted a concern about having OSA but only 8\% actually had a formal diagnosis of OSA and used CPAP. Fourteen percent of patients were rated as having a large tongue and $19 \%$ were rated as having a large uvula.

A total of 31 video clips of patients' soft palates were reviewed by the consulting expert otolaryngologist. His blinded ratings and those done in the operating room were identical in 28 of 31 cases (90\%), including 17 in which the retropalatal space was patent and 11 in which 
Table 1. Descriptive statistics.

\begin{tabular}{|c|c|c|c|c|c|}
\hline & Minimum & Maximum & Mean & Std. Deviation & $\begin{array}{l}\text { Correlation with Exp } \\
\text { Obstruction (Sig.) }\end{array}$ \\
\hline Age & 18 & 85 & 53.9 & 16.0 & 0.004 \\
\hline Gender (male coded 1 ) & 0 & 1 & 0.49 & 0.50 & 0.939 \\
\hline Excess skin in face & 0 & 1 & 0.09 & 0.29 & 0.193 \\
\hline Deep wrinkles in face & 0 & 1 & 0.16 & 0.36 & 0.114 \\
\hline Large lips & 0 & 1 & 0.09 & 0.29 & 0.735 \\
\hline Height (in) & 60 & 76 & 67.8 & 4.2 & 0.516 \\
\hline Weight (lbs) & 102 & 330 & 193.0 & 48.5 & 0.125 \\
\hline Neck circumference (in) & 13 & 20 & 15.7 & 1.7 & 0.013 \\
\hline Snoring & 0 & 2 & 0.93 & 0.80 & 0.000 \\
\hline OSA suspected & 0 & 1 & 0.23 & 0.43 & 0.001 \\
\hline OSA with CPAP & 0 & 1 & 0.08 & 0.27 & 0.632 \\
\hline Previous palate surgery & 0 & 1 & 0.01 & 0.11 & 0.471 \\
\hline Full beard & 0 & 1 & 0.07 & 0.25 & 0.347 \\
\hline Receding jaw & 0 & 1 & 0.02 & 0.15 & 0.645 \\
\hline Limited neck extension & 0 & 1 & 0.13 & 0.34 & 0.578 \\
\hline Thyromental distance $>6 \mathrm{~cm}$ & 0 & 1 & 0.94 & 0.23 & 0.489 \\
\hline Sub mental fat pad & 0 & 1 & 0.22 & 0.42 & 0.003 \\
\hline Upper edentulous & 0 & 1 & 0.20 & 0.40 & 0.295 \\
\hline Lower edentulous & 0 & 1 & 0.13 & 0.34 & 0.166 \\
\hline Friedman's tongue position & 1 & 4 & 1.91 & 0.82 & 0.004 \\
\hline High arched palate & 0 & 1 & 0.03 & 0.18 & 0.966 \\
\hline Big tongue & 0 & 1 & 0.14 & 0.35 & 0.000 \\
\hline Narrow pharynx & 0 & 1 & 0.01 & 0.11 & 0.000 \\
\hline Shallow nasopharynx & 0 & 1 & 0.04 & 0.20 & 0.834 \\
\hline Large uvula & 0 & 1 & 0.19 & 0.40 & 0.000 \\
\hline Large tonsils & 0 & 1 & 0.10 & 0.30 & 0.278 \\
\hline Can protrude lower jaw in front of upper & 0 & 1 & 0.86 & 0.35 & 0.257 \\
\hline Inspiratory obstruction head neutral & 0 & 1 & 0.58 & 0.50 & 0.471 \\
\hline Inspiratory obstruction with head tilted & 0 & 1 & 0.02 & 0.15 & 0.621 \\
\hline Expiratory obstruction & 0 & 1 & 0.34 & 0.48 & \\
\hline Expiratory obstruction with head tilted & 0 & 1 & 0.33 & 0.47 & 0.000 \\
\hline Soft palate lying on post pharynx & 0 & 1 & 0.33 & 0.47 & 0.000 \\
\hline Difficult intubation & 0 & 1 & 0.03 & 0.18 & 0.943 \\
\hline BMI & 18.2 & 47.2 & 29.4 & 6.4 & 0.025 \\
\hline
\end{tabular}

n = 90; OSA = Obstructive Sleep Apnea; CPAP = “continuous positive airway pressure”.

it was obstructed. The otolaryngologist rated three cases obstructed that the OR team rated as patent. The interrater reliability in these 31 cases provides assurance that inexperience or bias did not influence the ratings done in the operating room.

Fifty two of 90 patients (58\%) had inspiratory upper airway obstruction with the head in the neutral position. This problem was corrected by head tilt and chin lift in all but 2 patients, one of whom had tonsils so large they almost met in the midline and the other, limited neck extension due to prior cervical fusion. Both required an oral airway for ventilation; as a result, we could not de- 
termine if they also had expiratory obstruction. A diagnosis of expiratory upper airway obstruction was made in 30 of the remaining 88 patients (34\%) some with the head neutral (11) and some with the head tilted and chin lifted (19). Of the former, only one was cured of expiratory obstruction by subsequent head tilt and chin lift. In contrast, allowing the mouth to open cured expiratory obstruction in all patients and resolved breath stacking. Univariate correlation analysis identified a number of factors that were significantly related to expiratory airway obstruction (Table 1, last column). Multivariable regression analysis selected "large tongue", "large uvula" and "age in decades" as independent predictors of expiratory obstruction. The regression equation was statisticcally significant but accounted for only $45 \%$ of the observed variability. Expiratory airway obstruction was highly correlated with whether or not the soft palate and uvula was lying on the posterior pharyngeal wall $(\mathrm{P}<$ 0.001; Table 2).

\section{Discussion}

Evidence gathered in this quality improvement audit indicates that inspiratory upper airway obstruction during bag-mask ventilation was common but almost always corrected by head tilt and chin lift. On the other hand, expiratory upper airway obstruction during bag-mask ventilation with the mouth closed occurred in about a third of patients and was not corrected by head tilt and chin lift. Expiratory obstruction in supine patients occurs when the soft palate moves back against the posterior pharyngeal wall, forming a one-way "flap" valve that allows gas to enter the lungs through the nose but prevents gas from the lungs coming back out the nose. Expiratory obstruction masquerades as inspiratory obstruction because of breath stacking. The correct technique for bag-mask ventilation must include both careful observation for expiratory obstruction and intermittent removal of the mask which allows trapped gas to exit through the mouth. Inserting a properly-sized oral or nasal airway will also accomplish this goal.

Expiratory upper airway obstruction was recognized

Table 2. Correlation between occlusion of the retro-palatal space and breath stacking.

\begin{tabular}{ccccc}
\hline & Count & \multicolumn{2}{c}{} \\
\hline & & \multicolumn{2}{c}{ Breath stacking } & \multirow{2}{*}{ Total } \\
\cline { 2 - 4 } & & no & yes & \\
\hline \multirow{2}{*}{ Retro-palatal space occluded } & no & 55 & 3 & 58 \\
& yes & 3 & 27 & 30 \\
Total & & 58 & 30 & 88 \\
\hline
\end{tabular}

The association is highly significant: $\mathrm{P}<0.001$ by Chi Square analysis. during early work on cardiopulmonary resuscitation (CPR) by Safar and colleagues. In a letter to the editor, these authors commented (without providing any data) that CPR ventilation by firemen occasionally failed due to expiratory obstruction. They speculated that this obstruction was due to the soft-palate acting as a one-way "flap" valve [5]. Another report contains reference to unpublished observations of expiratory obstruction in 7 of 68 apneic subjects [6], which is a lower incidence than the current (unreferenced) textbook quote of "about 20\%" [4]. In contrast, our overall incidence was 34\%.

Opening the mouth is given as the treatment of choice when expiratory obstruction and breath stacking occurs $[4,6,8]$, and this maneuver was uniformly successful in our patients. A brief report suggests that head tilt, chin lift and/or elevating the head on a pillow should also correct the problem [9]; however, we found little evidence to support this concept; only one of our 11 subjects with expiratory obstruction when the head was in the neutral position improved with head tilt and chin lift.

Despite these early reports, clinical awareness of expiratory upper airway obstruction during bag mask ventilation seems to have vanished. Just the odd sentence or two about the issue appears in current textbooks $[4,8]$, and none of the recent studies of difficult mask ventilation during induction of anaesthesia even mentions expiratory obstruction as a possible mechanism [3,10-12]. Perhaps this is due to the previous use of black rubber masks which prevented observation of the mouth, or to the fact that breath stacking during expiratory obstruction mimics inspiratory obstruction because higher and higher bag pressures are required to achieve air entry. Both inspiratory and expiratory obstructions are easily fixed by insertion of an oral airway, so practitioners may have misdiagnosed the problem.

Our examination of the soft palate and uvula during laryngoscope insertion confirms that the soft palate was lying on the posterior pharyngeal wall in almost all patients with expiratory airway obstruction and that the retropalatal space was patent in almost all patients who did not develop expiratory airway obstruction.

This strong correlation in combination with the fact that gas can be delivered to the lungs through the nose but cannot escape through the nose confirms the "flap valve" nature of the expiratory obstruction. The fact that gas escapes from the lungs readily when the mouth is allowed to open indicates that the soft palate rather than the tongue is the site of expiratory obstruction. Concerns about the validity or accuracy of the use of visual inspection of the soft palate to help determine the site and mechanism of expiratory airway obstruction are not pertinent since method errors might contribute to a "negative" result but cannot explain the tight correlation between the patency of the soft palate and expiratory ob- 
struction that we observed. The flap-valve action of the soft palate should be confirmed by nasal endoscopy. Measurement of inspiratory and expiratory airflow via the nose could provide further confirmation of expiratory obstruction [13].

Observer error or distortion of the soft palate by the laryngoscope may account for the few cases in which expiratory obstruction occurred even though the retropalatal space appeared to be open. An alternative explanation is suggested by the selection of "large tongue" as an independent predictor of expiratory obstruction in the regression analysis. Perhaps posterior movement of the tongue during anaesthesia pushed the soft palate backwards, producing expiratory obstruction [14]. This effect would be reversed when the tongue was moved during visualization of the soft palate, allowing the retropalatal space to open.

The soft palate is quite mobile and previous studies using lateral neck $\mathrm{X}$-rays have shown that the distance between the soft palate and the posterior pharyngeal wall decreases during anaesthesia, to zero in some cases [15, 16]. Several previous studies have documented airway collapse at the level of the nasopharynx during sleep [17] and anaesthesia [18,19]; however these studies were done in subjects during spontaneous ventilation and the collapse occurred only when nasal pressure was suddenly reduced to low, even sub-atmospheric levels. Hence, the findings are not directly relevant to the current situation which involves positive pressure ventilation.

Our regression model with the clinical predictors "large tongue", "large uvula" and "decade" was statistically significant but able to account for only about $45 \%$ of the variability; this makes pre-operative prediction of expiratory obstruction an inexact science at best. Hence, practitioners engaged in bag-mask ventilation should have a high index of suspicion in all patients and look carefully for the signs of expiratory obstruction such as lack of chest fall and "flat line" expired $\mathrm{CO}_{2}$ traces. Unfortunately, changes in chest movement may be quite subtle as trapping develops and will be obscured if blankets cover the thorax. Further, capnography may not be available in settings such as the ward and field where patients undergo bag-mask ventilation. Hence, it seems reasonable to change our collective approach to bagmask ventilation to either always insert an oral (or nasal) airway or to intermittently allow the mouth to open. The latter approach is simple and avoids the problems associated with the use of oral and nasal airways such as trauma to the lips, tongue and teeth or epistaxis. An incorrectly sized or placed airway may also make ventilation more, rather than less, difficult. However, convinceing a provider who does not understand expiratory obstruction to release the mask to allow gas to escape from the mouth is more easily said than done. It is counter- intuitive to give up a good mask seal and take the mask off the face when having trouble getting gas into the patient. In fact, the characteristic response we have observed in this situation is to grip the face and mask tighter and squeeze the bag harder, which only worsens the problem.

The incidence of expiratory airway obstruction in our entire sample was $34 \%$, a finding that may, or may not translate to other patient populations. The incidence in patients who are simply asleep or unconscious but not paralyzed could conceivably be less since they likely have preserved tonic dilator muscle tone in the airway [20].

This audit was done to improve practitioner's ability to safely and effectively ventilate patients with a bag-mask system. The audit uncovered a high incidence of inspiratory upper airway obstruction with the head neutral that could be effectively managed with head tilt and chin lift in almost all cases. The audit also uncovered a 34\% incidence of expiratory airway obstruction that could not be fixed by head tilt and chin lift. Awareness of the phenomenon of expiratory upper airway obstruction and a change in procedure to allow the mouth to open from time to time should improve the safety of bag-mask ventilation.

\section{Acknowledgements}

We thank Drs. Paul M. Kempen, James V. Snyder, and Paul E. Bigeleisen for their thoughtful input.

\section{REFERENCES}

[1] M. P. Boidin, "Airway Patency in the Unconscious Patient,” British Journal of Anaesthesia, Vol. 57, No. 3, 1985, pp. 306-310. doi:10.1093/bja/57.3.306

[2] P. Safar, A. Escarraga and F. Chang, "Upper Airway Obstruction in the Unconscious Patient," Journal of Applied Physiology, Vol. 14, No. 5, 1959, pp. 760-764.

[3] M. R. Salem and A. Ovassapian, "Difficult Mask Ventilation: What Needs Improvement?” Anesthesia \& Analgesia, Vol. 109, No. 6, 2009, pp. 1720-1722. doi:10.1213/ANE.0b013e3181be0541

[4] J. P. McGee and J. S. Vender, "Nonintubation Management of the Airway: Mask Ventilation,” In: C. A. Hagberg, Ed., Benumof's Airway Management_Principles and Practice, Elsevier, Philadelphia, 2007, pp. 345-370. doi:10.1016/B978-032302233-0.50021-4

[5] P. Safar and J. Redding, “The 'Tight Jaw' in Resuscitation,” Anesthesiology, Vol. 20, 1959, pp. 701-702.

[6] S. Morikawa, P. Safar and J. Decarlo, "Influence of the Headjaw Position upon Upper Airway Patency,” Anesthesiology, Vol. 22, No. 2, 1961, pp. 265-270. doi:10.1097/00000542-196103000-00016

[7] M. Friedman, H. Tanyeri, R. M. La, R. Landsberg, K. Vaidyanathan, S. Pieri and D. Caldarelli, "Clinical Pre- 
dictors of Obstructive Sleep Apnea," Laryngoscope, Vol. 109, No. 12, 1999, pp. 1901-1907. doi:10.1097/00005537-199912000-00002

[8] W. H. Rosenblatt and W. Sukhupragarn, "Airway Management,” In: P. G. Barash, B. F. Cullen, R. K. Stoelting, M. K. Cahalan and C. M. Stock, Eds., Clinical Anesthesia, Lippincott and Williams \& Wilkins, New York, 2009, pp. 751-792.

[9] D. W. Galloway, "Upper Airway Obstruction by the Soft Palate: Influence of Position of the Jead, Jaw, and Neck," British Journal of Anaesthesia, Vol. 64, 1990, pp. 383384.

[10] M. El-Orbany and H. J. Woehlck, "Difficult Mask Ventilation,” Anesthesia \& Analgesia, Vol. 109, No. 6, 2009, pp. 1870-1880. doi:10.1213/ANE.0b013e3181b5881c

[11] S. Kheterpal, R. Han, K. K. Tremper, A. Shanks, A. R. Tait, M. O'Reilly and T. A. Ludwig, "Incidence and Predictors of Difficult and Impossible Mask Ventilation," Anesthesiology, Vol. 105, No. 5, 2006, pp. 885-891. doi:10.1097/00000542-200611000-00007

[12] O. Langeron, E. Masso, C. Huraux, M. Guggiari, A. Bianchi, P. Coriat and B. Riou, "Prediction of Difficult Mask Ventilation,” Anesthesiology, Vol. 92, No. 5, 2000, pp. 1229-1236. doi:10.1097/00000542-200005000-00009

[13] R. D. Warters, T. A. Szabo, F. G. Spinale, S. M. DeSantis and J. G. Reves, "The Effect of Neuromuscular Blockade on Mask Ventilation,” Anaesthesia, Vol. 66, No. 3, 2011, pp. 163-167. doi:10.1111/j.1365-2044.2010.06601.X

[14] Y. Liang, W. R. Kimball, R. M. Dacmarek, W. M. Zapol and Y. Jiang, "Nasal Ventilation Is More Effective than Combined Oral-Nasal Ventilation during Induction of
General Anesthesia in Adult Subjects,” Anesthesiology, Vol. 108, No. 6, 2008, pp. 998-1003. doi:10.1097/ALN.0b013e318174f027

[15] M. Mathru, O. Esch, J. Lang, M. E. Herbert, G. Chaljub, B. Goodacre and E. vanSonnenberg, "Magnetic Resonance Imaging of the Upper Airway: Effects of Propofol Anesthesia and Nasal Continuous Positive Airway Pressure in Humans," Anesthesiology, Vol. 84, No. 2, 1996, pp. 273- 279. doi:10.1097/00000542-199602000-00004

[16] P. R. Nandi, C. H. Charlesworth, S. J. Taylor, J. F. Nunn and C. J. Dore, "Effect of General Anaesthesia on the Pharynx,” British Journal of Anaesthesia, Vol. 66, No. 2, 1991, pp. 157-162. doi:10.1093/bja/66.2.157

[17] B. T. Woodson, "Expiratory Phayrngeal Airway Obstruction during Sleep: A Multiple Element Model,” The Laryngoscope, Vol. 113, No. 9, 2003, pp. 1450-1459. doi:10.1097/00005537-200309000-00004

[18] P. R. Eastwood, I. Szollosi, P. R. Platt and D. R. Hillman, "Collapsibility of the Upper Airway at Different Concentrations of Propofol Anesthesia," Anesthesiology, Vol. 103, No. 3, 2005, pp. 470-477. doi:10.1097/00000542-200509000-00007

[19] D. R. Hillman, J. H. Walsh, K. J. Maddison, P. R. Platt, J. P. Kirkness, W. J. Noffsinger and P. R. Eastwood, "Edolution of Changes in Upper Airway Collapsibility during Slow Induction of Anesthesia with Propofol," Anesthesiology, Vol. 111, No. 1, 2009, pp. 63-71. doi:10.1097/ALN.0b013e3181a7ec68

[20] G. B. Drummond, "Controlling the Airway: Skill and Science,” Anesthesiology, Vol. 97, No. 4, 2002, pp. 771-773. doi:10.1097/00000542-200210000-00002. 